\title{
Análisis de roles de equipo presentes y su implicación a través de la asignatura de ingeniería de proyectos. Caso de estudio en varios másteres de la Universidad Politécnica de Madrid. Analysis of present team roles and their implication through the subject of project engineering. Case of study: Several Masters of the Universidad Politécnica de Madrid.
}

\author{
M. Dolores Storch de Gracia ${ }^{1}$, Bernardo Llamas², Margarita Martínez Núñez ${ }^{3}$ \\ lola.storch@upm.es, bernardo.llamas@upm.es,margarita.martinez@upm.es
}

\author{
${ }^{1}$ Departamento Ingeniería Organización, \\ Administración de Empresas y Estadística \\ ETS Ingenieros Industriales.
}

\author{
Universidad Politécnica de Madrid \\ Madrid, España
}

2 Departamento Ingeniería Geológica
y Minera
ETS Ingenieros de Minas y Energía

Universidad Politécnica de Madrid Madrid, España

\author{
${ }^{3}$ Centro de Investigación en \\ Tecnologías Software y Sistemas \\ Multimedia para la Sostenibilidad \\ ETS de Ingeniería y Sistemas de \\ Telecomunicación \\ Universidad Politécnica de Madrid \\ Madrid, España
}

\begin{abstract}
Resumen-. El futuro mercado de trabajo de los actuales estudiantes de ingeniería demandará profesionales preparados para trabajar en equipo, una de las competencias transversales más valoradas por el mercado de trabajo. En este trabajo se presenta la aplicabilidad de la teoría de Belbin en la formación de equipos y la importancia de la presencia de los distintos roles en el desempeño del equipo. Además se presenta un caso de estudio a través de un ejercicio realizado en un master de ingeniero impartido por la Universidad Politécnica de Madrid, donde se han identificado roles clave para el desarrollo de proyectos innovadores desde una visión holística. Se pretende así estudiar el proceso de creación de equipos de trabajo y la influencia de este proceso en el éxito de los equipos de trabajo para después extender este análisis a otros másteres de la Universidad Politécnica de Madrid en la que los autores colaboran.
\end{abstract}

Palabras clave: competencias transversales; trabajo en equipo; roles.

Abstract- The future job market of current engineering students will require professionals prepared to work as a team, one of the transversal competences most valued by the labor market. This paper presents the applicability of Belbin's theory in the formation of teams and the importance of the presence of different roles in team performance. In addition, a case study is presented through an exercise carried out in an engineering master's degree taught by the Universidad Politécnica de Madrid, where key roles have been identified for the development of innovative projects from a holistic perspective. It is intended to study the process of creating work teams and the influence of this process on the success of work teams.

Keywords: transversal competences, teamwork, roles.

\section{INTRODUCCIÓN}

En un mundo cada vez más complejo y rico en información, el valor de la colaboración está creciendo en importancia.
Cada vez más se está trabajando por equipos en lugar de por individuos.

El motivo de crear un equipo surge por la necesidad de responder a una necesidad específica (proyecto, grupo de trabajo, etc...) y en muchas ocasiones son equipos temporales formados por personas que pertenecen a otros departamentos y deben contribuir de manera puntual en el equipo. Es más, dependiendo de la estructura organizativa que tenga implantada la organización para el desarrollo de proyectos, estos equipos serán más o menos temporales (B. Llamas et al 2016).

Tanto si los miembros de equipos de innovación pertenecen de manera exclusiva a un Departamento de Innovación como si provienen de otros departamentos, la característica principal que los define es el grado de excelencia que se espera de su desempeño.

El Dr. Meredith Belbin, de la Universidad de Cranfield, obtuvo una respuesta convincente y ampliamente documentada que ayudó de manera crucial a entender el funcionamiento de los grupos y como HACER UN TRABAJOR mejor.

El descubrimiento fundamental de Belbin es que todos los miembros de un equipo de gestión tienen un doble rol. El primer rol, el papel funcional, es intrínseco a su puesto (ingeniero de producción, contable, ejecutivo de marketing, etc...) pero el segundo rol, que denominaremos el "rol de equipo", es mucho menos evidente y sin embargo, todos somos un poco conscientes desde que entramos en un equipo nuestra afinidad o nuestra preferencia a la hora de trabajar y de cómo ejercemos nuestro trabajo - y estas características propias del individuo serán siempre constantes para el individuo en cualquier equipo o proyecto. 
Belbin sugiere que cada persona tiene un doble rol según se muestra en la Figura 1.

\section{Rol funcional}

El trabajo para el que me
han escogido, basado en
mis habilidades
experiencias y aptitudes

Qué hacemos......
Rol de equipo

Nuestra tendencia a comportarnos, contribuciones e interrelaciones en un cierto modo particular

Cómo lo hacemos......
Figura 1. Roles funcionales y de equipo

A la hora de reclutar personas en un equipo de trabajo, además de las aptitudes de Belbin, deben seleccionarse personas capaces de complementar los puntos fuertes y compensar debilidades, establecer objetivos SMART ${ }^{1}$, evaluar el entorno interno y externo y es necesario adaptar el estilo de liderazgo (teoría liderazgo situacional, o teoría Belbin).

Mientras que todos los equipos están formados por individuos, no todos los grupos de individuos forman un equipo.

Belbin propone que el resultado del trabajo en equipo es efectivo cuando sus miembros trabajan como "jugadores de equipo” - donde cada jugador entiende cuán importante es para todos cooperar y trabajar con un objetivo común. Cuando todos hacen su trabajo bien, esto incrementa las metas que el equipo puede conseguir. Es clave para esto que cada persona sepa lo que aporta al equipo (desde el punto de vista funcional y de comportamiento).

Un rol está definido por seis factores: personalidad, habilidad mental, actuales valores y motivaciones, limitaciones, experiencia y aprendizaje del rol.

La indicación es que, sin ignorar o descuidar al individuo, debemos prestar mucha mayor atención a los equipos: a su SELECCIÓN, DESARROLLO y FORMACIÓN pero sobre todo a su PSICOLOGÍA, MOTIVACIÓN, COMPOSICIÓN y COMPORTAMIENTO ¿Pero cómo?

El primer problema que enfrentamos es que la psicología ha estado tradicionalmente orientada a individuos y el conocimiento de equipos de éxito es muy escaso.

$\mathrm{Al}$ enumerar las cualidades del buen gestor vemos que muchas cualidades son excluyentes: debe ser enérgico pero sensible a las personas, dinámico pero paciente, comunicarse bien, pero escuchar atentamente, tomar decisiones pero reflexionar, etc. Todo a la vez no es posible y aunque lo fuera, aunque encontráramos a esta maravillosa persona, podría irse un día.

Sin embargo, un equipo si puede reunir estas cualidades y no suelen irse todos a la vez y por esta razón el éxito de la gestión se debe fundamentalmente al equipo. Un equipo puede atesorar las cualidades necesarias, puede renovarse reclutando nuevas personas y acumular gran cantidad de experiencia compartida y puede estar en varios lugares a la vez. Todos hemos visto como una persona con gran éxito en un equipo

${ }^{1}$ Objetivo SMART: específico, medible, alcanzable, realista y temporal. cuando ha cambiado de equipo no ha lucido tanto; hemos visto equipos eficaces que se destruyen por el ascenso de individuos sin considerar al resto del equipo o como equipos que producían una calidad y cantidad de trabajo mucho mayores que la suma del trabajo que los individuos hubieran podido producir individualmente.

Tras diversas investigaciones, Belbin identificó nueve roles de equipo que los integrantes de los equipos suelen adoptar: Coordinador, Impulsor, Cerebro, Evaluador, Implementador, Investigador de recursos, Cohesionador, Finalizador y Especialista. En la Figura 2 se muestran los nueve roles y su característica principal y su caracterización principal: aquellos reflexivos, focalizados en la acción o sociales.

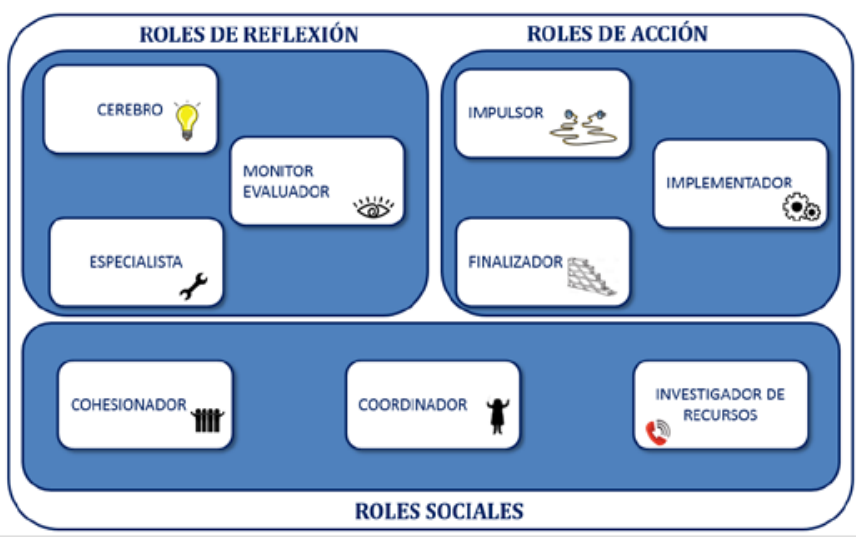

Figura 2. Roles de equipo y orientación principal

Un resumen de las principales características de cada rol sería el siguiente:

Coordinador: Es el encargado de coordinar esfuerzos para llegar a los objetivos. Establece la agenda, es tranquilo, confiado y responsable de mantener el equilibrio del equipo. Es el líder social (estable, dominante, extrovertido).

Impulsor: es el emprendedor de tareas e influye en las decisiones del equipo; Está dispuesto a ser impopular si el trabajo lo exige. Es el líder de tareas (ansioso, dominante, extrovertido).

Cerebro: genera ideas y desarrolla formas innovadoras de solucionar problemas; altamente creativas y buenas resolviendo problemas de manera poco convencional. (Muy inteligente, dominante, introvertido).

Evaluador: es el analizador desapasionado, analítico y objetivo; puede molestar a sus compañeros de equipo con sus críticas, pero casi nunca se equivoca (muy inteligente, estable, introvertido).

Implementador: Es el organizador práctico. Trabaja duro para convertir ideas en hechos, puede molestar a sus compañeros de equipo porque es reacio al compromiso. Necesita estabilidad (Estable, controlado).

Investigador de recursos: a menudo astuto y entusiasta, es el más relajado; encuentra los recursos necesarios y cala enseguida cualquier oposición. (Estable, dominante, extrovertido).

Cohesionador: Es el comunicador interno más activo, soporte del grupo sociable; une el equipo y ayuda a solventar 
cualquier conflicto interpersonal y profesional que se produzca en el seno del equipo. (Estable, extrovertido, poco dominante).

Finalizador: Se preocupa de lo que puede fallar, su máxima preocupación es el orden y las fechas y compromisos. Decidido y comprometido, aunque se le puede considerar interesado en terminar el trabajo cueste lo que cueste (ansioso, introvertido).

Especialista: proporciona experiencia técnica en áreas clave; puede molestar a los demás por centrarse única y exclusivamente en su área de especialización.

Más detalles de estos perfiles se pueden obtener en el capítulo dedicado a las personas del (B. Llamas et al, 2017).

Las conclusiones principales Belbin fueron las siguientes:

a. La ausencia de un papel debilita el equipo, pero también si hay demasiados con el mismo rol.

b. Un rol de equipo muestra nuestra "tendencia a comportarnos, contribuir e interrelacionarlos de una manera particular"

c. Si hay menos de 9 personas se adoptan varios roles (principal y secundario) mientras que en grandes equipos se suelen formar subgrupos donde aparecen estos roles en cada uno de ellos

d. El conocimiento de los roles en equipos establecidos ayuda menos que en los nuevos equipos donde la combinación de roles se ha mostrado muy efectiva

e. La construcción de equipos a través del equilibro de papeles es más importante en áreas de trabajo bajo presión o de acción rápida así como en ambientes donde la creatividad es necesaria (I+D especialmente, pero en general también proyectos de ingeniería)

f. Los equipos formados por mayoría de gente muy inteligente generalmente fracasan.

\section{CONTEXTO}

Los estudiantes de ingeniería deben adquirir, además de conocimientos específicos, formación en competencias transversales, entre las que destaca el trabajo en equipo (Mazadiego et al, 2017).

De esta forma, el ingeniero no solo adquiere conocimientos propios de su área de especialización, sino que también adquiere una serie de competencias que faciliten su incorporación al mercado laboral, y su éxito en su desarrollo profesional.

La Escuela Técnica-Superior de Ingenieros de Minas y Energía de la Universidad Politécnica de Madrid (ETSIMEUPM) imparte varios grados y másteres, destacando la excelencia docente mediante las acreditaciones otorgadas por la acreditadora ABET (Accreditation Board for Engineering and Technology).-a la mayor parte de los programas docentes que se imparten en la misma.

Como proceso de mejora continua de la formación docente, se trabajan en el desarrollo de herramientas docentes que permita impulsar la formación en competencias transversales, como es el trabajo en equipo. Son muchos los enfoques y proyectos de innovación educativa abordados por el equipo docente, sin embargo, en este caso se muestra un recurso enfocado hacia la adquisición de competencias de trabajo en equipo en el área de ingeniería de proyectos, y en concreto, el proceso crítico de creación de equipos de trabajo.

En este marco, al conocido recurso didáctico de fomentar el trabajo en equipo mediante trabajos grupales, se añade en las asignaturas de ingeniería de proyectos y evaluación de proyectos y gestión del riesgo la formación en roles de equipo, comportamiento del individuo frente al trabajo (Teoría Jung) y liderazgo situacional (Teoría Blanchard) (Llamas \& Storch, 2017).

El conocimiento de los roles de equipo, las tendencias de cada integrante y del resto del equipo ayudarán a obtener resultados óptimos en su desempeño como estudiantes, pero luego también como profesionales.

\section{DESCRIPCIÓN}

Una de las competencias principales en el desempeño profesional es la capacidad del individuo para trabajar en equipo ya que cada vez es más necesario poder trabajar de manera coordinada con otras personas.

La teoría de Belbin sostiene que el éxito del equipo vendrá determinado por el equilibrio en la presencia de distintos roles.

Para valorar el rol principal de cada integrante del equipo se utilizó un cuestionario basado en el trabajo de Belbin que es suficiente para destacar la presencia de los distintos roles en un individuo.

Este cuestionario de Autopercepción para miembros de equipos consta de siete secciones y en cada sección se invita a que se distribuyan en cada sección un total de 10 puntos entre las afirmaciones que mejor describan tu comportamiento.

En casos extremos podrían repartirse entre todas las afirmaciones o se podrían otorgar 10 puntos a una sola afirmación.

Como resultado se obtiene el porcentaje de presencia de cada rol en el individuo y se puede concluir que rol o roles principales tendrá presente.

Realizando el cuestionario de Belbin a todos los miembros del equipo se obtiene la información de presencia de roles en el mismo así como el acumulado de cada rol como suma de todas las contribuciones de los integrantes a ese rol en concreto.

En el marco de la asignatura de "Evaluación de Proyectos y Gestión del Riesgo" se propuso a todos los alumnos que realizaran el cuestionario individual e identificara el equipo de trabajo.

Los equipos se crearon espontáneamente, sin ninguna directriz por parte del profesorado (Spencer, 1989), si bien la formación académica de todos los integrantes era la misma y el tamaño de cada equipo era: Equipo 1 (5 miembros), Equipo 2 (6 miembros), Equipo 3 (5 miembros), Equipo 4 (3 miembros).

Se consideró realizar el cuestionario de Belbin a cuatro grupos de trabajo de la Asignatura de "Evaluación de proyectos y gestión del riesgo”, con el fin de analizar el comportamiento de los individuos y el rendimiento del trabajo grupal (Aguileta-Guemez, 2017).

Los grupos de trabajo se habían formado para desarrollar un proyecto innovador que debía presentarse ante un grupo de 
inversores ficticio compuesto por directivos de empresas de sectores que habitualmente contrata ingenieros de minas (Ejercicio del Inversionista) (B. Llamas et al, 2017).

Conviene destacar que bajo las premisas anteriores, será vital el funcionamiento del grupo y la presencia de roles equilibrados para conseguir resultados óptimos en el trabajo a realizar y la exposición del mismo ante el grupo de inversionistas.

\section{RESUltados}

Los resultados del cuestionario de Belbin se muestran en la Figura 3, donde se han recogido los resultados de todos los miembros y su rol principal apilando los resultados totales.

Destaca el rol de implementador, acusado en todos los estudiantes y el especialista, un resultado muy consistente con el perfil de los estudiantes dado el gran contenido aplicado y práctico (implementador) de los estudios de Ingeniería y su alto grado de especialización (especialista).

Los roles de cerebro e investigador de recursos son los menos destacados en la muestra en estudio. Estos últimos roles son esenciales en el desarrollo de proyectos innovadores, puesto que el cerebro aporta la solución innovadora (competencia: creatividad), mientras que el investigador de recursos aporta la capacidad de comunicación y de relacionarse con el entorno externo.

Los resultados que alcanzaron los equipos en el ejercicio del inversionista apoyan la tesis de la necesidad de equipos equilibrados, y en concreto, la necesidad de individuos que conecten con el exterior y defiendan la idea en una etapa vital en todo proyecto: la aprobación del mismo y su financiación. De entre todos los equipos analizados, aquel que presentó fuertes inversiones y un menor rol de investigador de recursos fue el menos valorado por el ejercicio del inversionista.

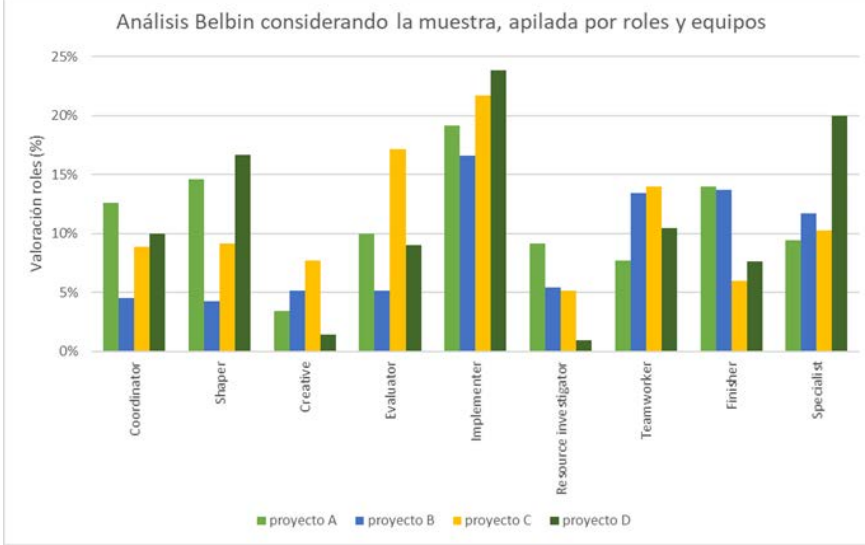

Figura 3. Resultados obtenidos de Roles presentes para cuatro equipos según teoría y cuestionario Belbin.

\section{CONCLUSIONES}

El trabajo en equipo es una de las competencias fundamentales para el desempeño de la ingeniería. En un entorno cada vez más competitivo como consecuencia de la globalización y el entorno tecnológico cambiante.
Adicionalmente, otras competencias genéricas, como creatividad y comunicación son necesarias considerando las diferentes etapas en el marco lógico de un proyecto.

La generación de equipos de trabajo es un proceso crítico en el éxito de cualquier proyecto, y no solo el conocimiento es clave en la configuración de un equipo exitoso.

En un entorno docente, como es el caso del ejercicio aquí expuesto, donde se ha dado la responsabilidad a los estudiantes para configurar los equipos se ha demostrado que no siempre es efectiva.

Para los futuros profesionales, hoy alumnos, es importante el que conozcan los roles predominantes por su personalidad, y de esta forma, buscar su desarrollo y acomodo en los equipos de trabajo de forma que estos individuos sean más efectivos.

Las conclusiones principales del análisis de los roles de equipo a la vista también de los resultados obtenidos son:

- El rol de Cerebro está muy poco desarrollado en todos los equipos, especialmente acusado este déficit en los proyectos A y D. Esto indica un déficit formativo en la capacidad de resolver e innovar problemas de ingeniería (competencia crea o resuelve). Además, estos equipos obtuvieron una menor calificación en sus proyectos, debido a la ausencia de integrantes con este rol.

- El rol más presente, tanto a nivel individual como acumulado es el rol de Implementador. Bajo la interpretación de los autores, esto se explica debido a la educación de las ingenierías que fomentan en el alumnado la orientación a resultados y las competencias específicas como aplica y experimenta.

- Considerando las diferentes fases de un equipo (identificación de necesidades, búsqueda de ideas, formulación de planes, establecimiento de organización del equipo y desarrollo del mismo), los equipos que tengan Coordinadores y Motores tendrán más éxitos en las fases iniciales de los proyectos (lanzan los proyectos, diseño conceptual y básico) mientas que los Implementadores y Finalizadores serán fundamentales en las últimas fases (los rematadores de proyectos, ingeniería de detalle, constructiva y ejecución del proyecto). En este sentido el equipo A está muy equilibrado mientas que el equipo $\mathrm{B}$ acusará déficit en el lanzamiento del proyecto y los proyectos C y D tienen unos resultados discretos.

- El rol de Investigador de recursos es crítico para el éxito del equipo, especialmente en el proceso de aprobación y obtención de financiación. Los resultados en los 4 equipos son discretos y especialmente acusada su ausencia en el equipo D que presenta dificultades para desenvolverse en el entorno exterior $\mathrm{y}$, como consecuencia dificultad para alcanzar la aprobación de la financiación (especialmente notorio en aquellos proyectos donde el presupuesto sea elevado)... Se constata su necesidad cuanto mayor recurso externo sea necesario.

- El rol de Especialista está presente en todos los equipos, algo lógico si tenemos en cuenta el perfil técnico de todos los miembros.

Esta metodología educativa, podría aplicarse en contextos formativos de máster de ingeniería, donde se busca una formación especializada y una, cada vez más, cercana con el 
mercado, ayudando a diseñar equipos eficientes no solo considerando los roles técnicos, sino que también trabajando y equilibrando equipos según su rol de equipo.

El proceso de creación de equipos eficientes pasa por el análisis de roles técnicos y de equipo, y no tanto por un proceso de integración de forma libre como ha sido el caso en el estudio aquí presentado. Este estudio de roles de equipo (teoría Belbin) debería ser completado con análisis de cómo el individuo se enfrenta al trabajo (teoría Jung) y de los diferentes estilos de liderazgo (teoría Blanchard).

De esta forma, futuros trabajos del equipo de investigación se centrarán en ahondar en el proceso constitutivo de los equipos de trabajo, dirigiendo el proceso de creación. De esta forma se podrá estudiar con mayor detalle el impacto de los roles aplicado a la ingeniería de proyectos.

Finalmente, conviene recoger que dos de los cuatro proyectos presentados y recogidos en este estudio fueron seleccionados en la primera fase del concurso de ideas actuaupm de la UPM (edición 14)).

El caso de estudio aquí presentado podría ser reproducido en otras ramas educativas en ingeniería, algo que los autores ya están considerando para ampliar su estudio a otras escuelas en el curso 2017/2018.

\section{REFERENCIAS}

ABET, Accreditation Board for Engineering and Technology, Inc http://www.abet.org/, acceso el 28 de mayo de 2017.

Actúaupm:

http://www.upm.es/Investigacion/innovacion/CreacionE mpresas/Servicios/Competicion_Creacion_Empresas acceso el 18 de junio de 2017.
Agencia Nacional de Evaluación de la Calidad y Acreditación, ANECA, www.aneca.es, acceso el 28 de mayo de 2017.

Aguileta-Guemez, Antonio A. (2017) Exploring the influence of Belbin roles in the quality code generated by students in a course on software engineering. Revista Educación en Ingeniería, 12(23), 93-100

Kagan S. (1989). The structural approach to cooperative learning. Educational Leadership, 47(4), 12'

Llamas B., Pous J., Storch de Gracia M.D. (2016). Apuntes de la asignatura de ingeniería de proyectos. Madrid, España Fundación Gómez-Pardo. ISBN: 978-84-6088312-8.

Llamas B., Storch de Gracia M.D. (2017). Apuntes de la asignatura de evaluación de proyectos y gestión del riesgo. De una idea a un proyecto. Madrid, España Fundación Gómez-Pardo. ISBN: 78-84-617-7988-8.

Llamas B., Storch de Gracia M.D., Mazadiego L.F. (Ed) (2017). Key Issues for Management of Innovative Projects. Intech Open. ISBN 978-953-51-3468-8, Print ISBN 978-953-51-3467-1.

Llamas B., Storch de Gracia M.D., Mazadiego L.F., Pous J., Alonso J. (2017). Assessing creativity as a critical competence in engineering. Case of study in mines engineering degree. Revista de educación. Forthcoming

Mazadiego L.F., Llamas B., Ortega M.F., Storch de Gracia M.D., Hernandez L., Merino P., Cámara A. (2017). Valoración de las competencias por parte de las empresas que contratan a los egresados de la Escuela Técnica Superior de Ingenieros de Minas y Energía (UPM). Revista de Educación. Forthcoming 